International Journal of Environmental Research and

Public Health

ISSN 1660-4601

www.mdpi.com/journal/ijerph

Article

\title{
Beneficial Effect of Higher Dietary Fiber Intake on Plasma HDL-C and TC/HDL-C Ratio among Chinese Rural-to-Urban Migrant Workers
}

\author{
Quan Zhou ${ }^{1, \dagger}$, Jiang Wu ${ }^{2, \dagger}$, Jie Tang ${ }^{1}$, Jia-Ji Wang ${ }^{1}$, Chu-Hong Lu ${ }^{1}$ and Pei-Xi Wang ${ }^{1, *}$ \\ 1 Faculty of Preventive Medicine, School of Public Health, Guangzhou Medical University, \\ Guangzhou, 195\# West Dongfeng Road, Guangzhou 510182, China; \\ E-Mails: joan_zq@126.com (Q.Z.); gytanjie@163.com(J.T.); wjia@163.com (J.W.); \\ chuhon688@163.com (C.L.) \\ 2 Shenzhen Baoan City Center Hospital, 6 Xiyuan Road, Baoan District, Shenzhen 518102, China; \\ E-Mail: WuJiang731@163.com
}

$\dagger$ These authors contributed equally to this work.

* Author to whom correspondence should be addressed; E-Mail: peixi001@163.com; Tel.: +86-20-8134-0186; Fax: +86-20-8134-0196.

Academic Editor: Paul B. Tchounwou

Received: 12 March 2015 / Accepted: 23 April 2015 / Published: 29 April 2015

\begin{abstract}
Research has shown that high-dose supplemental dietary fiber intake has beneficial effects on cardiovascular risk factors. To clarify such a relationship, we examined the association between daily dietary fiber intake and plasma lipids using a cross-sectional design including 1034 (M 502, F 532) rural-to-urban workers in China. We found a dose-response relationship between increased dietary fiber intakes and increase of HDL cholesterol in male workers. There was also a dose-response relationship between increased dietary fiber intake and decreased total cholesterol to HDL cholesterol (TC/HDL-C) ratio in both male and female workers, after adjusting for potential confounders ( $p$ for trend, all $p<0.05$ ). When the average dietary fiber intake increased from less than $18 \mathrm{~g}$ /day to over $30 \mathrm{~g}$ /day, the average HDL cholesterol level increased by $10.1 \%$, and the TC/HDL-C ratio decreased by $14.4 \%$ for males $(p=0.020)$ and by $11.1 \%$ for females $(p=0.048)$. In conclusion, higher daily dietary fiber consumption is associated
\end{abstract}


with beneficial effect on cholesterol for rural-to-urban workers in China, suggesting its potential beneficial effect on decreasing the risk of cardiovascular diseases.

Keywords: dietary fiber; high-density lipoprotein cholesterol; total cholesterol to highdensity lipoprotein cholesterol ratio

\section{Introduction}

Cardiovascular disease (CVD) remains the leading cause of mortality worldwide and caused about 17.5 million death in 2012 [1]. The World Health Organization (WHO) estimates that 75\% of cardiovascular mortality could be decreased with appropriate lifestyle changes; and this is the biggest challenge presented by the various guidelines on CVD prevention [2].

One strategy that has been attempted for this purpose is increasing the dietary fiber (DF) intake. As the residue of plant food resistant to hydrolysis by human alimentary enzymes, DF is a non-digestible carbohydrate and includes a heterogeneous mixture of polysaccharides and lignin [3], offering potential health benefits to humans [4]. Since the first finding that dietary fiber has some beneficial effects on ischaemic heart disease at the 1970s [5], lots of observational and experimental studies have explored the relationship between dietary fiber or fiber-rich foods and cardiovascular diseases or cardiovascular diseases risk factors, like obesity, hypertension and dyslipidemia [6-8].

Total cholesterol to high-density lipoprotein cholesterol (TC/HDL-C) ratio is a high discriminatory power index for coronary heart disease. High TC/HDL-C ratio has been intensively used as a predictor of CVD. Previous studies have reported a beneficial effect of DF intake on TC/HDL-C ratio, but the average DF intake in those studies was about $48 \mathrm{~g} /$ day [9], much higher than the average Chinese DF intake of $18 \mathrm{~g} /$ day [10].

High-density lipoprotein (HDL) plays a role in reverse cholesterol transport, reveals that HDL may suppress cholesterol accumulation in the peripheral tissues. Both epidemiological and basic science studies have shown an inverse association between the concentration of plasma HDL cholesterol (HDLC) and the risk of developing atherosclerotic CVD [11]. According to results of meta-analyses, whether DF intake has beneficial effects on HDL-C could not be determined [12]. Yet the result of clinical trials performed in Asian populations suggested that DF intake may help people maintain or increase their plasma HDL-C values [13].

The differences of DF intake amount may partially explain the different results. The "Dietary Guidelines for Chinese Residents" recommends a DF intake of $30 \mathrm{~g} /$ day [14], higher than that of WHO recommendation (25 g/day) [15]. To date, there is little research evaluating whether this amount of DF intake has any beneficial effects on blood lipid profiles in a Chinese population. Hence, this study was undertaken to evaluate the association of DF intake with plasma lipids in rural-to-urban migrant workers. 


\section{Experimental Section}

\subsection{Study Subjects}

This is a sub-study of the 2013 rural-to-urban migrant workers study performed in the Shenzhen-Dongguan economic zone of China, which was approved by the Research Ethics Board of Guangzhou Medical University (Guangzhou, China). The detailed methodology of this study is briefly described below. Three factories (a textile manufacturer, a furniture assembler and a camera assembler) were approached and they agreed to the implementation of the study protocol. Migrant workers between 18 and 59 years of age in the three factories were sampled by a cluster randomization method. One third of workshops in the three factories were randomly sampled. All subjects working in the sampled workshops were recruited ( $n=2315$ rural-to-urban migrant workers). Among them, all subjects $(n=1228)$ from the Dongguan camera assembler were invited to take a nutritional survey. Of these, 1127 participated in the present study (response rate, 91.8\%). Subjects who were taking lipids or blood glucose-lowering drugs and those with a history of cardiovascular diseases $(n=31)$ were excluded. Finally, a total of 1096 participants were included for the present study.

\subsection{Data Collection}

\subsubsection{Questionnaire Interview}

Information on sociodemographic data, health history, general risk factors of CVD, physical activities, current dietary intakes, and female reproductive history were collected by trained interviewers using a face-to-face interviewing of structured questionnaire. Questionnaire interview was conducted prior to laboratory assay.

\subsubsection{Dietary Assessment}

Participants completed a three-day diet record, in which they noted all foods and drinks during two weekdays and one weekend day [16]. Food models in the reference portion sizes and standard tablewares were provided as visual aids to help participants weigh the amount of foods and drinks they consumed. If weighing was not possible, participants were instructed to estimate the amount of foods and drinks they consumed by using standard household measures (e.g., spoon, glass, cup, etc.). Intake of dietary fiber and other nutrients was calculated based on the Chinese Food Composition Table (data of soluble and insoluble fiber are not discriminated) [17]. Energy-adjusted DF intake (g/1000 kcal/day) was calculated using the energy density method [18]. In the present study, individuals who reported unrealistic energy intake ( $<500$ or $>5000 \mathrm{kcal} /$ day, $n=62$ ) were excluded from the final data analyses.

\subsubsection{Anthropometric and Blood Pressure Measurement}

The height and weight of participants were measured to the nearest $0.5 \mathrm{~cm}$ and $0.1 \mathrm{~kg}$ respectively. Body mass index (BMI) was then calculated as weight $(\mathrm{kg}) /$ height $(\mathrm{m})^{2}$. Waist circumference (WC) was measured half way between the lowest rib margin and the iliac crest, and hip circumference was measured at the level of the greater trochanters. Measurements of WC and hip circumference were performed twice and the 
average values were used for analyses. Waist to hip ratio (WHR) was then calculated. Two consecutive measurements of blood pressure were taken from the right arm after subjects had been sitting for at least $10 \mathrm{~min}$. Systolic blood pressure (SBP) and diastolic blood pressures (DBP) were recorded to the nearest 2 $\mathrm{mm} \mathrm{Hg}$. If the two systolic or diastolic blood pressures recorded differed $\geq 4 \mathrm{mmHg}$, a third measurement was made. The average value of the two blood pressures was used for analyses. SBP and DBP were defined as the point of the appearance (Korotkoff I) and disappearance (Korotkoff V) of Korotkoff sounds, respectively.

\subsubsection{Laboratory Assay}

The 12-h fasting venous blood was collected with vacuum tubes containing EDTA for lipids analysis. Plasma was separated after centrifugation at $1500 \times \mathrm{g}$ for $15 \mathrm{~min}$ at $4{ }^{\circ} \mathrm{C}$ within $2 \mathrm{~h}$ and stored at $-80{ }^{\circ} \mathrm{C}$ till tests. All samples were analyzed in a single batch within 5 days to minimize laboratory variability. Plasma TC, TG, HDL-C and LDL-C were measured with colorimetric methods using commercial kits (Biosino Biotechnology Company Ltd., Beijing, China) by an automated analyzer (A25 Biosystem, Barcelona, Spain). The coefficients of variation for lipid measurements were $2.3 \%$ (at $4.50 \mathrm{mmol} / \mathrm{L} \mathrm{TC}), 5.8 \%($ at $1.77 \mathrm{mmol} / \mathrm{L}$ TG), $4.3 \%$ (at $1.28 \mathrm{mmol} / \mathrm{L}$ HDL-C), and 3.1\% (at $3.29 \mathrm{mmol} / \mathrm{L}$ LDL-C). TC/HDL-C was calculated as TC $(\mathrm{mmol} / \mathrm{L}) / \mathrm{HDL}-\mathrm{C}(\mathrm{mmol} / \mathrm{L})$. Laboratory assay was conducted by two staffs which were not involved in the questionnaire interview and did not know the exposure status of the subjects.

\subsection{Statistical Analysis}

Results were presented as mean (S.D.). Mean dietary intakes were corrected for within-person variation by means of the multiple source method (MSM) [19]. Based on the average DF intake of Chinese ( $18 \mathrm{~g} /$ day) and the recommended daily dietary fiber intake in China (30 g/day) participants were categorized into either $>30 \mathrm{~g} /$ day (high DF intake group, HI group), between $18 \mathrm{~g} /$ day and $30 \mathrm{~g} /$ day (median DF intake group, MI group), or $<18 \mathrm{~g}$ /day (low DF intake group, LI group). Comparisons of characteristics among the three groups were performed using either one-way ANOVA or $\chi^{2}$ test. The statistical significances of pairwise comparisons were corrected by Bonferroni test. Mean differences in plasma lipids and blood glucose among the three dietary fiber groups were compared using analysis of covariance (ANCOVA), after adjusting for age, BMI, waist circumference, smoking, total dietary energy, dietary fat and protein, and energy from saturated fat. Assumptions of tests were examined and confirmed prior to analyses. Two-way analysis of covariance was used to examine the interaction between sex and the three levels of dietary fiber intake on plasma lipids. All analyses were conducted with SPSS for Windows (version 13.0, SPSS, Inc., Chicago, IL, USA). A two-sided $p$-value of less than 0.05 was considered as statistically significant.

\section{Results and Discussion}

\subsection{Results}

The characteristics of the 1034 participants are shown in Table 1. The mean (S.D.) ages of female and male participants were 36.7 (8.1) and 36.4 (7.5) years, respectively. There was no significant difference in BMI between male and female workers, significant differences were found in waist circumstance, hip circumstance and waist to hip ratio. 
Table 1. Characteristics (mean \pm S.D.) of the 1034 rural-to-urban migrant workers.

\begin{tabular}{|c|c|c|c|}
\hline & $\operatorname{Men}(n=134)$ & Women $(n=272)$ & $p$-Value \\
\hline Age (year) & $36.7 \pm 8.1$ & $36.4 \pm 7.5$ & 0.628 \\
\hline Weight (kg) & $60.8 \pm 9.1$ & $55.8 \pm 8.9$ & $<0.001$ \\
\hline Height $(\mathrm{cm})$ & $161.2 \pm 7.0$ & $152.9 \pm 6.2$ & $<0.001$ \\
\hline Body mass index $\left(\mathrm{kg} / \mathrm{m}^{2}\right)$ & $24.1 \pm 3.2$ & $23.8 \pm 3.3$ & 0.235 \\
\hline BMI category $(\%)$ & & & 0.043 \\
\hline Underweight & 0.5 & 2.7 & \\
\hline Normal weight & 53.9 & 57.1 & \\
\hline Overweight & 34.7 & 29.0 & \\
\hline Obesity & 10.4 & 11.3 & \\
\hline Waist circumference $(\mathrm{cm})$ & $77.2 \pm 8.1$ & $75.5 \pm 8.8$ & 0.006 \\
\hline Hip circumference $(\mathrm{cm})$ & $89.0 \pm 5.7$ & $90.1 \pm 6.4$ & 0.016 \\
\hline Waist to hip ratio & $0.9 \pm 0.06$ & $0.8 \pm 0.1$ & $<0.001$ \\
\hline Systolic blood pressure (mmHg) & $119.8 \pm 15.7$ & $115.3 \pm 13.9$ & $<0.001$ \\
\hline Diastolic blood pressure (mmHg) & $73.6 \pm 13.1$ & $70.6 \pm 9.8$ & $<0.001$ \\
\hline Smoking & & & $<0.001$ \\
\hline Non-smoker (\%) & 40.5 & 98.9 & \\
\hline Ex-smoker (\%) & 6.3 & 0.0 & \\
\hline Current-smoker (\%) & 53.2 & 1.1 & \\
\hline \multicolumn{4}{|l|}{ Diet intake } \\
\hline Energy (kcal/day) & $1874.8 \pm 694.9$ & $1657.0 \pm 638.8$ & $<0.001$ \\
\hline Protein (g/day) & $62.5 \pm 34.4$ & $54.0 \pm 32.2$ & 0.001 \\
\hline Total fat (g/day) & $70.3 \pm 36.1$ & $64.2 \pm 31.6$ & 0.120 \\
\hline Saturated fatty acids (\%kcal) & $14.6 \pm 12.8$ & $15.4 \pm 11.7$ & 0.344 \\
\hline Polyunsaturated fatty acids (\%kcal) & $9.7 \pm 6.7$ & $10.6 \pm 7.4$ & 0.079 \\
\hline Energy adjusted dietary fiber (g/1000 kcal) & $9.5 \pm 6.7$ & $10.0 \pm 6.2$ & 0.298 \\
\hline Total dietary fiber (g/day) & $15.5 \pm 8.0$ & $14.5 \pm 7.0$ & 0.061 \\
\hline Total dietary fiber category & & & 0.313 \\
\hline$\sim 18 \mathrm{~g} /$ day & 73.9 & 78.6 & \\
\hline$\sim 30 \mathrm{~g} /$ day & 20.5 & 17.1 & \\
\hline$\geq 30 \mathrm{~g} /$ day & 5.6 & 4.3 & \\
\hline \multicolumn{4}{|l|}{ Serum biomarkers } \\
\hline Total cholesterol (mmonl/L) & $4.6 \pm 0.9$ & $4.4 \pm 0.8$ & 0.006 \\
\hline HDL cholesterol (mmol/L) & $1.4 \pm 0.3$ & $1.5 \pm 0.3$ & $<0.001$ \\
\hline LDL cholesterol (mmol/L) & $2.2 \pm 0.5$ & $2.1 \pm 0.5$ & 0.001 \\
\hline Triglycerides $(\mathrm{mmol} / \mathrm{L})$ & $1.5 \pm 0.9$ & $1.0 \pm 0.8$ & $<0.001$ \\
\hline TC to HDL-C ratio & $3.4 \pm 0.9$ & $3.1 \pm 0.7$ & $<0.001$ \\
\hline Blood Glucose (mmol/L) & $5.0 \pm 0.9$ & $5.1 \pm 0.9$ & 0.427 \\
\hline
\end{tabular}

The mean (S.D.) dietary fiber intakes in female and male workers were 15.5 (8.0) and 14.5 (7.0) g/day, and the mean energy-adjusted DF intakes were 9.5 (6.7) and 10.0 (6.2), respectively. The majority of subjects consumed less than the average amount of DF (76.2\%), including $73.9 \%$ males and $78.6 \%$ females. Only 5\% subjects consumed the recommended daily intake level including $5.6 \%$ males and $4.3 \%$ females. Males had significantly higher intake of energy $(p<0.001)$ and protein $(p=0.001)$ than females. Furthermore, over-weight (OW) and obese males consumed less total DF compared with under- weight 
(UW) and normal-weight (NW) peers (Figure 1). No significant differences were observed in total and energy-adjusted DF intakes among females of different BMI categories.

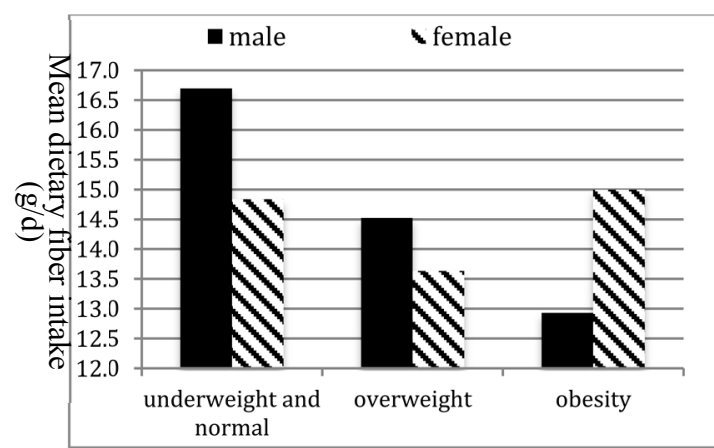

(a)
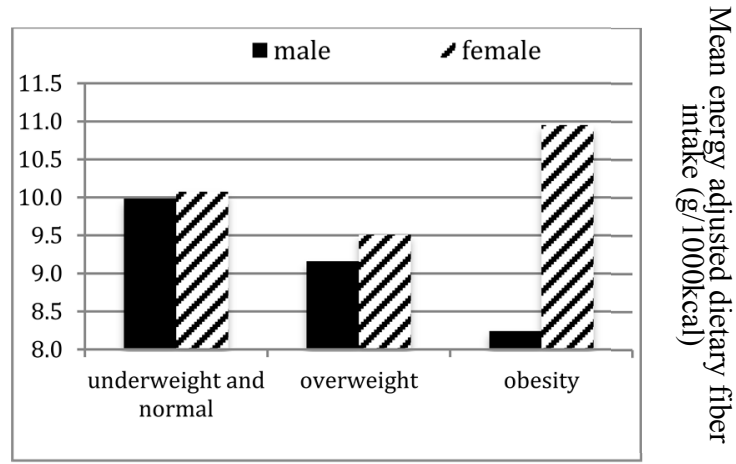

(b)

Figure 1. Mean (a) total and (b) energy-adjusted dietary fiber intakes by BMI category stratified by gender of rural-to-urban participants.

Univariate analysis showed a dose-response relationship between increased HDL-C and increased DF intake in male workers ( $p$ for trend 0.004). For male workers the mean HDL-C was increased by $11.3 \%$ in HI group comparing with that in LI group. And a negative correlation between DF intake and TC/HDL-C ratios in male ( $p$ for trend 0.004 ) and female ( $p$ for trend 0.022 ) workers were found, the mean TC/HDL-C ratio was decreased by $17.5 \%$ for male workers and $12.7 \%$ for females in HI group comparing with LI group. No significant differences of TC, LDL-C, BP, triglycerides and blood glucose among the three DF intake groups were observed in both male and female workers (all $p>0.05$ ). Higher DF intake was also associated with higher intakes of total protein and total fat, but there was significantly lower daily intake of total carbohydrates in this sample of individuals (Table 2).

Table 2. Comparison of means of parameter by three DF intake Groups.

\begin{tabular}{|c|c|c|c|c|c|c|c|c|c|}
\hline & \multicolumn{2}{|c|}{ Low } & \multicolumn{2}{|c|}{ Mid } & \multicolumn{2}{|c|}{ High } & \multirow{2}{*}{$\begin{array}{l}\% \text { Difference } \\
\text { (high vs. low) }\end{array}$} & \multirow{2}{*}{$\begin{array}{c}p \text { for } \\
\text { one-way } \\
\text { ANOVA } \\
\end{array}$} & \multirow{2}{*}{$\begin{array}{l}p \text { for } \\
\text { linear } \\
\text { trend } \\
\end{array}$} \\
\hline & Mean & S.D. & Mean & S.D. & Mean & S.D. & & & \\
\hline \multicolumn{10}{|l|}{$\operatorname{Men}(n=502)$} \\
\hline Systolic BP (mmHg) & 120.2 & 16.2 & 119.9 & 15.4 & 120.8 & 14.9 & 0.5 & 0.971 & 0.873 \\
\hline Diastolic BP (mmHg) & 73.6 & 13.2 & 74.7 & 12.3 & 76.7 & 11.8 & 4.0 & 0.511 & 0.306 \\
\hline $\begin{array}{l}\text { Total cholesterol } \\
(\mathrm{mmol} / \mathrm{L})\end{array}$ & 4.6 & 0.9 & 4.6 & 1.0 & 4.4 & 0.9 & -4.1 & 0.599 & 0.379 \\
\hline $\begin{array}{l}\text { HDL cholesterol } \\
(\mathrm{mmol} / \mathrm{L})\end{array}$ & 1.3 & 0.3 & 1.4 & 0.3 & $1.5 *$ & 0.3 & 11.3 & 0.014 & 0.006 \\
\hline $\begin{array}{l}\text { LDL cholesterol } \\
(\mathrm{mmol} / \mathrm{L})\end{array}$ & 2.2 & 0.5 & 2.2 & 0.5 & 2.0 & 0.5 & -8.8 & 0.301 & 0.135 \\
\hline TC/HDL-C & 3.5 & 0.9 & 3.4 & 0.8 & $3.0 *$ & 0.5 & -17.5 & 0.011 & 0.004 \\
\hline Triglycerides (mmol/L) & 1.5 & 0.8 & 1.6 & 1.0 & 1.6 & 0.8 & 7.4 & 0.841 & 0.613 \\
\hline Blood Glucose (mmol/L) & 5.0 & 0.9 & 5.0 & 0.7 & 4.8 & 0.6 & -3.7 & 0.642 & 0.347 \\
\hline Age (year) & 36.5 & 8.3 & 37.5 & 7.7 & 36.2 & 8.9 & -1.1 & 0.630 & 0.841 \\
\hline Body mass index $\left(\mathrm{kg} / \mathrm{m}^{2}\right)$ & 24.5 & 3.3 & $23.2 *$ & 2.5 & $21.8 *$ & 2.8 & -12.2 & $<0.001$ & $<0.001$ \\
\hline Waist circumference $(\mathrm{cm})$ & 77.7 & 8.9 & 77.7 & 8.9 & 75.7 & 6.9 & -2.6 & 0.613 & 0.323 \\
\hline
\end{tabular}


Table 2. Cont.

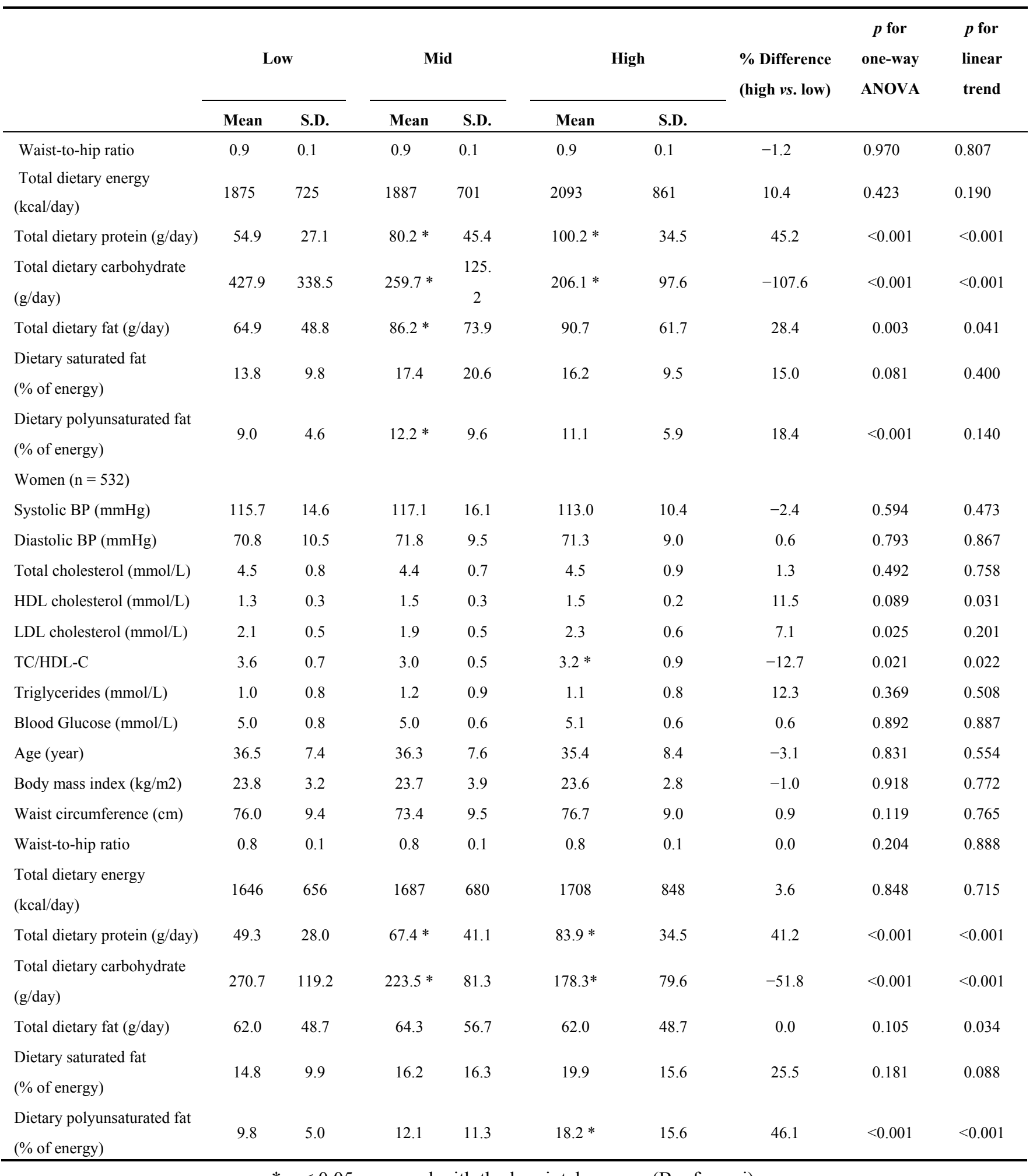

$* p<0.05$ compared with the low intake group (Bonferroni).

Analysis of covariance was conducted to examine whether there was an independent association between daily DF intake and blood lipids and glucose after adjusting for potential confounders, such as age, BMI, waist circumference, smoking, total dietary energy, intakes of protein, fat, saturated fatty acids and polyunsaturated fatty acids. Similar associations were observed (Table 3 ). The mean value of HDL-C was $10.1 \%$ higher in male workers $(p=0.02)$, and the TC/HDL-C ratio was $14.4 \%(p=0.028)$ and $11.1 \%$ 
$(p=0.048)$ lower in male and female workers, respectively, when comparing the HI group with the LI group of DF intake (Table 3).

\subsection{Discussion}

In this cross-sectional study, we found that increased DF intake was significantly associated with an increase plasma HDL-C level in male workers after adjusting for confounding factors (e.g., age, BMI, waist circumference, smoking, total dietary energy, intakes of protein, fat, saturated fatty acids and polyunsaturated fatty acids), as well as for a decreased TC/HDL-C ratio in male and female workers. HDL-C was increased by $10.1 \%$ for male and TC/HDL-C ratio was decreased by $14.4 \%$ and $11.1 \%$ for males and females respectively, when daily DF intake was higher than $30 \mathrm{~g} /$ day.

HDL is thought to function as a sterol transporter that facilitates the movement of sterols from peripheral cells to the liver. High HDL-C level is associated with a reduced risk of coronary heart disease [20,21]. Recently, Schaffer et al. [22] reported that the relative risk of coronary artery disease was 1.48 (95\% CI, 1.37-1.6) in subjects with HDL-C $<32 \mathrm{mg} / \mathrm{dl}$ as compared with the highest quintile of HDL-C ( $>47 \mathrm{mg} / \mathrm{dl})$ after adjusting for potential confounders in a cohort study. Our findings also implied that higher daily DF intake might be associated with a lower risk of CVD in Chinese rural-to-urban male workers.

Table 3. Covariable-adjusted means of parameters by three DF intake Groups.

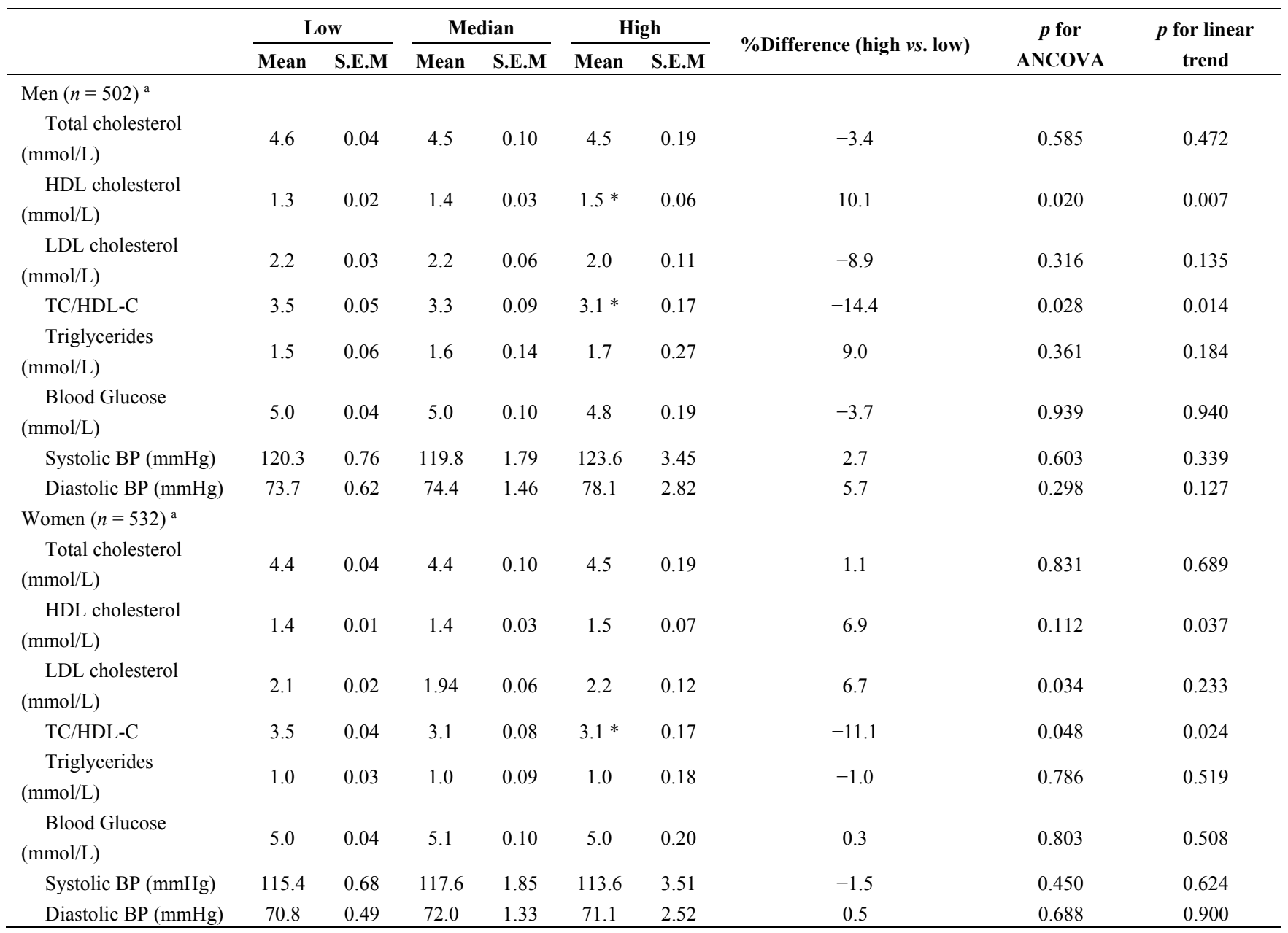

a Adjusted for age, BMI, waist circumference, smoking status and energy, protein, saturated fat, polyunsaturated fat, and carbohydrate intake; $* p<0.05$ compared with the low intake group (Bonferroni). 
To date, studies examining the association between DF intake and risk of CVD have yielded inconsistent results. A sub-study of the Japan Collaborative Cohort Study including 58,730 adults aged 40-79 y showed that covariates-adjusted CVD risk was 0.79 (95\% CI: $0.61-0.98$ ) in subjects consuming a high DF of $>12.6 \mathrm{~g} / \mathrm{d}$ as compared with those consuming a low intake of $<7.8 \mathrm{~g} /$ day [23]. Yet a prospective study in the EU reported no statistically significant difference in CVD risk among quintile groups of DF intake (from $14.1 \mathrm{~g} /$ day to $38.3 \mathrm{~g} /$ day) after a 14.3-year follow-up of 31,036 females [24]. Our findings seem to support a favorable association between DF intake and a lower CVD risk. The reason for the differences among these observational studies was unclear. The different sources of DF in ethnic foods may contribute in part to the contradictory results. Hopping et al. [25] reported that the protective effects of dietary fiber on the development of diabetes differed by ethnic group according to consumed foods, which may be a plausible theory applying to cardiovascular diseases as well.

It has been established that high plasma TC, LDL-C, TG, TC/HDL-C and low HDL-C increase risk of CVD. Randomized controlled trials in Asia had found that high-dose DF supplemental generally had a favorable effect on serum lipid profiles. In the present study, TC, LDL-C and TG were not associated with dietary fiber intake. We observed a significant difference of HDL-C among the three groups of daily DF intake in rural-to-urban male workers. We also observed significant differences of TC/HDL-C ratios in both male and female workers.

Studies evaluating the relationship between dietary fiber and HDL-C have given inconsistent results. Studies performed in Asian populations supported that dietary fiber was beneficially associated with HDL$\mathrm{C}$, while randomized controlled trials in patients with hypertension [26,27] suggested that increased intake of soluble dietary fiber was not associated with increased HDL-C. Another trial using refined konjac meal as source of dietary fiber yielded a similar result [28]. A meta-analysis [12] showed that the use of barley did not significantly alter HDL-C $(p=0.07)$ in healthy and hypercholesterolemic individuals. A recent trial performed in China investigated the impact of oat consumption on cholesterol levels in Chinese adults with mild to moderate hypercholesterolemia, the result of which indicated that the HDL-C level was unchanged in oat group which is rich in dietary fiber after 6 weeks intervention [13]. In the present study, we found that a dietary fiber intake of over $30 \mathrm{~g} /$ day might be beneficial to males for improving their plasma HDL-C.

TC/HDL-C ratio is more sensitive in reflecting the morbidity and severity of CVD than individual lipid levels. A randomized trial performed by Reid et al. [29] reported that subjects who consumed onethird cup per day of psyllium-containing cereal and were advised to increase soluble fiber intake to over $10 \mathrm{~g}$ /day for 26 weeks gained a 4.6\% TC/HDL-C ratio reduction. Similar results were found in another clinical trial, in which 4 weeks of hydroxypropylmethyl cellulose consumption $(5 \mathrm{~g} /$ day) resulted in significant reductions of TC/HDL-C ratio along with TC, non-HDL-C and LDL-C [30]. Maki et al. [31] provided $3 \mathrm{~g} /$ day of $\beta$-glucan from whole-grain ready-to-eat oat cereal to overweight and obese adults and observed reductions in TC and LDL-C as early as week 4. Consistent with the meta-analysis by Kelly et al. [32], which indicated that oat consumption is associated with lower TC ( $p=0.005)$ and LDL-C concentrations ( $p=0.008)$, Ballesteros et al. [9] also reported a beneficial effect of high DF intake (48 g/day) on plasma TC, LDL-C and TG in adult men. In the present study, TC/HDL-C ratio was negatively associated with total dietary fiber intake. However, we did not observe significant association between dietary fiber intake and TC, LDL-C and TG, which is possibly due to limited sample size or a large random error in fiber intake measurement. 
Interestingly in the current study we detected that OW and obese males consumed less total DF compared with UW and NW males. Although DF has been used in the prevention or treatment of obese in children and adults [33], conflicted results about the association between dietary fiber intake and body weight were reported. Previous findings [34] suggested that DF intake during puberty might not affect the concurrent development of body fat percentage or BMI. However, Pal et al. [35] reported that after supplement with fiber for 12 weeks, subjects had a significantly reduced body weight and BMI along with great improvements in serum biomarkers of CVD. Recently, Lin et al. [36] reported that OW and obese females consumed less total and energy-adjusted DF compared with UW and NW peers. Another study [37] suggested that total DF intake was significantly lower in Swiss OW boys, but not girls. In present study the tendency of total DF intake throughout the weight groups shows that obese female workers had the highest consumption of DF. However, differential misreporting (e.g., more underreporting among the obese) could bias those results.

Some limitations of this study need to be considered. The major limitation of this study is the crosssectional study design, in which causal relationship is hard to be identified due to no clear time-sequence relationship between the relevant exposures and outcomes. Moreover, inverse causal relationships are often observed if the exposures could be changed due to the outcomes of interest. In this study, we excluded all subjects who had previously confirmed conditions which might change their dietary habits, such as dislipidemia, diabetes, hypertension, as well as CVD. Inverse causal relationship between dietary fiber and cardiovascular risk factors could thus be excluded in our study.

Another limitation is that the 24-h dietary recall method does not allow accurate assessments of infrequently consumed foods. In order to correct for such errors, nutrient intakes were corrected for within-person variability by applying the MSM method. Furthermore, we computed dietary fiber intake in the present study according to the China Food Composition Table, which so far don't discriminate soluble from insoluble fiber. Therefore, the beneficial effect of dietary fiber on HDL-C is not clear, which may be elucidated in our future study.

Additionally, in the current study subjects were rural-to-urban workers from local factories rather than a population-based random sample. These subjects tended to have younger age, lower educational status and income than general population. However, no significant associations between dietary intake and education and economic status were observed. Therefore, these social economic differences between our subjects and the general population would be unlikely to significantly affect the DF-blood lipid association. Finally, it should be mentioned that the small sample size and differential recall by weight status may affect the result of this study.

\section{Conclusions}

Higher dietary fiber consumption is associated with increased plasma HDL-C in Chinese rural-tourban male workers and with decreased TC/HDL-C ratio in Chinese rural-to-urban male and female workers. Our findings suggest dietary fiber may be beneficial for the prevention of cardiovascular diseases

\section{Acknowledgements}

The present study was funded by Guangzhou 121 Talents Program, China. 


\section{Author contributions}

Quan Zhou and Jiang $\mathrm{Wu}$ were responsible for the data analyses and the drafting of the manuscript. All authors contributed to conception and the interpretation of the results. All authors read and approved the final manuscript.

\section{Conflicts of Interest}

The authors declare that they have no competing interests.

\section{References}

1. World Health Organization. The 10 Leading Causes of Death in the World, 2000 and 2012; World Health Organization: Geneva, Switzerland, 2014.

2. World Health Organization. Global Status Report on Noncommunicable Diseases 2010; World Health Organization: Geneva, Switzerland, 2011.

3. EFSA Panel on Dietetic Products and Allergies (NDA). Scientific opinion on dietary reference values for carbohydrates and dietary fibre. EFSA J. 2010, 8, doi:10.2903/j.efsa.2010.1462.

4. Joint FAO/WHO expert consultation on diet NatPoCD. Diet, Nutrition, and the Prevention of Chronic Diseases; WHO Technical Report series; WHO: Geneva, Switzerland, 2003; pp. 91634-91663.

5. Trowell, H. Ischemic heart disease and dietary fiber. Am. J. Clin. Nutr. 1972, 25, 926-932.

6. Truswell, A.S. Cereal grains and coronary heart disease. Eur. J. Clin. Nutr. 2002, 56, 1-14.

7. Van Horn, L.; McCoin, M.; Kris-Etherton, P.M.; Burke, F.; Carson, J.A.; Champagne, C.M.; Karmally, W.; Sikand, G. The evidence for dietary prevention and treatment of cardiovascular disease. J. Am. Diet Assoc. 2008, 108, 287-331.

8. Mente, A.; de Koning, L.; Shannon, H.S.; Anand, S. A systematic review of the evidence supporting a causal link between dietary factors and coronary heart disease. Arch. Internal Med. 2009, 169, 659-669.

9. Ballesteros, M.N.; Cabrera, R.M.; Saucedo, M.S.; Yepiz-Plascencia, G.M.; Ortega, M.I.; Valencia, M.E. Dietary fiber and lifestyle influence serum lipids in free living adult men. J. Am. Coll. Cardiol. 2001, 20, 649-655.

10. Zhai, F.; He, Y.; Hu, Y.; Wang, Z.; Yu, W.; Yang, X. The status of dietary fiber intake of Chinese people in 2002. Acta Nutrimenta Sinica 2005, 27, 444-447.

11. Emerging Risk Factors Collaboration; Di Angelantonio, E.; Sarwar, N.; Perry, P.; Kaptoge, S.; Ray, K.K.; Thompson, A.; Wood, A.M.; Lewington, S.; Sattar, N.; et al. Major lipids, apolipoproteins, and risk of vascular disease. JAMA 2009, 302, 1993-2000.

12. Yanai, H.; Katsuyama, H.; Hamasaki, H.; Abe, S.; Tada, N.; Sako, A. Effects of carbohydrate and dietary fiber intake, glycemic index and glycemic load on HDL metabolism in Asian populations. J. Clin. Med. Res. 2014, 6, 321-326.

13. Zhang, J.; Li, L.; Song, P.; Wang, C.; Man, Q.; Meng, L.; Kurilich, A. Randomized controlled trial of oatmeal consumption versus noodle consumption on blood lipids of urban Chinese adults with hypercholesterolemia. Nutr. J. 2012, 11, doi:10.1186/1475-2891-11-54. 
14. Dietary Guidelines for Chinese Residents; Chinese Nutrition Society: Beijing, China, 2009.

15. Möller, J.R. Dietary Fibre-Codex Definition and Methods of Analysis; A White Paper from FOSS; FOSS: Hilleroed, Denmark, 2011.

16. Deriemaeker, P.A.D.; Hebbelinck, M.; Clarys, P. Validation of a 3-day diet diary: Comparison with a 7-day diet diary and a FFQ. Med. Sci. Sports Exerc. 2006, 38, S328.

17. Yang, Y.X.; Wang, G.Y.; Pan, X.C. China Food Composition 2002; Peking University Medical Press: Beijing, China, 2002.

18. Stookey, J.D. Energy density, energy intake and weight status in a large free-living sample of Chinese adults: Exploring the underlying roles of fat, protein, carbohydrate, fiber and water intakes. Eur. J. Clin. Nutr. 2001, 55, 349-359.

19. Multiple Source Method; Department of Epidemiology of the German Institute of Human Nutrition Potsdam-Rehbrucke: Nuthetal, Germany, 2009.

20. Kitamura, A.; Iso, H.; Naito, Y.; Iida, M.; Konishi, M.; Folsom, A.R.; Sato, S.; Kiyama, M.; Nakamura, M.; Sankai, T.; et al. High-density lipoprotein cholesterol and premature coronary heart disease in urban Japanese men. Circulation 1994, 89, 2533-2539.

21. Mertens, A.; Holvoet, P. Oxidized LDL and HDL: Antagonists in atherothrombosis. FASEB J. Off. Publ. Fed. Am. Soc. Exp. Biol. 2001, 15, 2073-2084.

22. Schaffer, A.; Verdoia, M.; Barbieri, L.; Aprami, T.M.; Suryapranata, H.; Marino, P.; Luca, G.D.; Novara Atherosclerosis Study Group. High-density lipoproteins and coronary artery disease: A single-center cohort study. Angiology 2014, 65, 696-702.

23. Eshak, E.S.; Iso, H.; Date, C.; Kikuchi, S.; Watanabe, Y.; Wada, Y.; Wakai, K.; Tamakoshi, A.; JACC Study Group. Dietary fiber intake is associated with reduced risk of mortality from cardiovascular disease among Japanese men and women. J. Nutr. 2010, 140, 1445-1453.

24. Threapleton, D.E.; Greenwood, D.C.; Burley, V.J.; Aldwairji, M.; Cade, J.E. Dietary fibre and cardiovascular disease mortality in the UK women's cohort study. Eur. J. Epidemiol. 2013, 28, 335-346.

25. Fujii, H.; Iwase, M.; Ohkuma, T.; Ogata-Kaizu, S.; Ide, H.; Kikuchi, Y.; Idewaki, Y.; Joudai, T.; Jirakawa, Y.; Uchida, K.; et al. Impact of dietary fiber intake on glycemic control, cardiovascular risk factors and chronic kidney disease in Japanese patients with type 2 diabetes mellitus: The Fukuoka Diabetes Registry. Nutr. J. 2013, 12, doi:10.1186/1475-2891-12-159.

26. Singh, R.B.; Rastogi, S.S.; Singh, R.; Ghosh, S.; Niaz, M.A. Effects of guava intake on serum total and high-density lipoprotein cholesterol levels and on systemic blood pressure. Am. J. Cardiovas. 1992, 70, 1287-1291.

27. Singh, R.B.; Rastogi, S.S.; Singh, N.K.; Ghosh, S.; Gupta, S.; Niaz, M.A. Can guava fruit intake decrease blood pressure and blood lipids? J. Hum. Hypertens. 1993, 7, 33-38.

28. Zhang, M.Y.; Huang, C.Y.; Wang, X.; Hong, J.R.; Peng, S.S. The effect of foods containing refined Konjac meal on human lipid metabolism. Biomed. Environ. Sci. 1990, 3, 99-105.

29. Reid, R.; Fodor, G.; Lydon-Hassen, K.; D’Angelo, M.S.; McCrea, J.; Bowlby, M.; Difrancesco, L. Dietary counselling for dyslipidemia in primary care: Results of a randomized trial. Can. J. Diet. Pract. Res. 2002, 63, 169-175. 
30. Maki, K.C.; Carson, M.L.; Miller, M.P.; Anderson, W.H.; Turowski, M.; Reeves, M.S.; Kaden, V.; Dicklin, M.R. Hydroxypropylmethylcellulose lowers cholesterol in statin-treated men and women with primary hypercholesterolemia. Eur. J. Clin. Nutr. 2009, 63, 1001-1007.

31. Maki, K.C.; Beiseigel, J.M.; Jonnalagadda, S.S.; Gugger, C.K.; Reeves, M.S.; Farmer, M.V.; Kaden, V.N.; Rains, T.M. Whole-grain ready-to-eat oat cereal, as part of a dietary program for weight loss, reduces low-density lipoprotein cholesterol in adults with overweight and obesity more than a dietary program including low-fiber control foods. J. Am. Diet Assoc. 2010, 110, 205-214.

32. Kelly, S.A.; Summerbell, C.D.; Brynes, A.; Whittaker, V.; Frost, G. Wholegrain cereals for coronary heart disease. Cochrane Database Syst. Rev. 2007, doi:10.1002/14651858.

33. Wanders, A.J.; van den Borne, J.J.; de Graaf, C.; Hulshof, T.; Jonathan, M.C.; Kristensen, M.; Mars, M.; Schols, H.A.; Feskend, E.J. Effects of dietary fibre on subjective appetite, energy intake and body weight: A systematic review of randomized controlled trials. Obes. Rev. Off. J. Int. Assoc. Stud. Obes. 2011, 12, 724-739.

34. Cheng, G.; Karaolis-Danckert, N.; Libuda, L.; Bolzenius, K.; Remer, T.; Buyken, A.E. Relation of dietary glycemic index, glycemic load, and fiber and whole-grain intakes during puberty to the concurrent development of percent body fat and body mass index. Am. J. Epidemio 2009, 169, 667-677.

35. Pal, S.; Khossousi, A.; Binns, C.; Dhaliwal, S.; Ellis, V. The effect of a fibre supplement compared to a healthy diet on body composition, lipids, glucose, insulin and other metabolic syndrome risk factors in overweight and obese individuals. Br. J. Nutr. 2011, 105, 90-100.

36. Lin, Y.; Huybrechts, I.; Vereecken, C.; Mouratidou, T.; Valtuena, J.; Kersting, M.; Gonzalez-Gross, M.; Bolca, S.; Warnberg, J.; Cuenca-Garcia, M.; et al. Dietary fiber intake and its association with indicators of adiposity and serum biomarkers in European adolescents: The Helena study. Eur. J. Nutr. 2014, doi:10.1007/s00694-014-0756-2.

37. Aeberli, I.; Kaspar, M.; Zimmermann, M.B. Dietary intake and physical activity of normal weight and overweight 6 to 14 year old swiss children. Swiss Med. Wkly. 2007, 137, 424-430.

(C) 2015 by the authors; licensee MDPI, Basel, Switzerland. This article is an open access article distributed under the terms and conditions of the Creative Commons Attribution license (http://creativecommons.org/licenses/by/4.0/). 